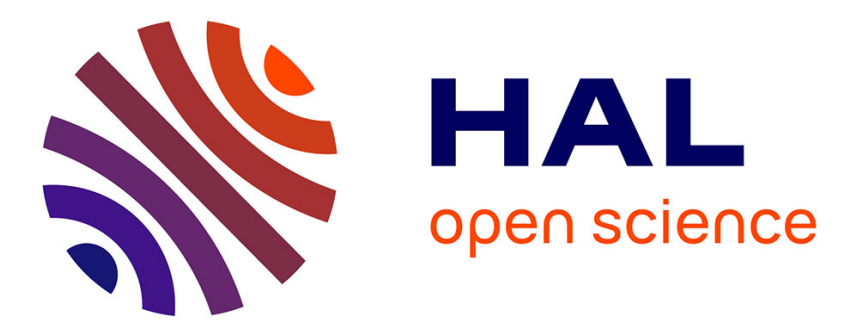

\title{
Friction and instability of steady sliding: squeal of a rubber/glass contact
}

\author{
Didier Vola, Michel Raous, J.A.C a C Martins
}

\section{To cite this version:}

Didier Vola, Michel Raous, J.A.C a C Martins. Friction and instability of steady sliding: squeal of a rubber/glass contact. International Journal for Numerical Methods in Engineering, 1999, 46 (10), pp.1699-1720. hal-03178204

\section{HAL Id: hal-03178204 \\ https://hal.science/hal-03178204}

Submitted on 24 Mar 2021

HAL is a multi-disciplinary open access archive for the deposit and dissemination of scientific research documents, whether they are published or not. The documents may come from teaching and research institutions in France or abroad, or from public or private research centers.
L'archive ouverte pluridisciplinaire HAL, est destinée au dépôt et à la diffusion de documents scientifiques de niveau recherche, publiés ou non, émanant des établissements d'enseignement et de recherche français ou étrangers, des laboratoires publics ou privés. 


\title{
REFERENCE :
}

D. VOLA, M. RAOUS, J.A.C. MARTINS, Friction and instability of steady sliding: squeal of a rubber/glass contact, International Journal for Numerical Methods in

Engineering, 46, 1999, pp. 1699-1720.

\section{Friction and instability of steady sliding: squeal of a rubber/glass contact}

\author{
D. Vola and M. Raous \\ Equipe Mécanique et Modélisation du Contact, \\ Laboratoire de Mécanique et d'Acoustique, Marseille, France
}

\author{
J.A.C. Martins \\ Departamento de Engenharia Civil and ICIST, \\ Instituto Superior Técnico, Lisboa, Portugal
}

\begin{abstract}
The dynamic instability of steady sliding states of finite dimensional frictional contact systems with non-linear elastic behavior is analyzed. An algorithm for the computation of those steady sliding states and a sufficient condition for their instability, based on the resolution of a generalized eigenvalue problem, are presented. Flutter instabilities due to the non-associative character of the Coulomb friction law are shown to occur for a finite element model of a rubberlike waist seal sliding on a glass window that is known to generate squeal noise. The consequences of those flutter instabilities are assessed by computing various finite element dynamic solutions in the neighborhood of steady sliding.
\end{abstract}

\section{INTRODUCTION}

This work is part of a research effort devoted to the study of instability phenomena in frictional contact problems. More specifically, it deals with the generation of noise during the sliding of a deformable body on a rigid obstacle. The case of a rubber/glass contact 
between a waist seal and a car window is discussed.

In a previous work ${ }^{15}$, the dynamic stability of a finite dimensional frictional contact system with linear elastic behavior was discussed. The theory was applied to study the possible origines for the occurrence of jumps on the time evolution of the friction force and the propagation of stress waves along the contact zone, in the course of the sliding of a polyurethane block on a flat araldite plate ${ }^{15,22,26,27}$, . A generalization of this work to finite dimensional nonlinear elastic systems in frictional contact with curved obstacles may be found in ${ }^{14}$.

In the present work, a nonlinear material behavior is considered (finite elasticity, Mooney Rivlin strain energy), and stability analyses are performed for steady sliding equilibrium states. A fixed point algorithm on the tangential contact stresses is used to compute the steady sliding solutions in a range of values of the coefficient of friction, which is the essential parameter in the stability analyses. The occurrence of dynamically unstable steady sliding states (existence of smooth growing dynamic solutions with perturbed initial conditions arbitrarily close to steady sliding) is predicted by solving appropriate generalized eigenvalue problems. The two matrices in these problems are finite element matrices depending on the frictional contact conditions. When a flutter instability is detected, its consequences are studied by performing dynamic analyses of the system behavior. The formulation of the finite dimensional dynamic problem is done in terms of differential measures, leading to a numerical method that handles the velocity jumps on the solution.

Finite dimensional stability analyses of steady sliding states were also performed in ${ }^{13,17}$, for some metallic bodies, under the assumption of rigid or linear elastic behavior, and adopting a nonlinear compliance law for the normal behavior of the interface. Although many aspects of the present work are quite distinct from those of ${ }^{13,17}$ (geometry and boundary conditions of the bodies, material and interface properties, and also the computational algorithms) it is clear that the cause for the flutter instabilities detected in ${ }^{13,17}$ and in the present paper is the same : the non-symmetry (non-self-adjointness) of Coulomb's friction law. Furthermore, despite all those distinctions, the consequences of the flutter instabilities found in the present paper have some qualitative features that clearly resemble some of those discussed in ${ }^{13}$.

In section 2, the continuum formulation of the dynamic problem in finite elasticity with frictional unilateral contact is presented. Section 3 is devoted to formulate and approximate the finite dimensional dynamical problem. Section 4 focuses on the direct computation of the steady sliding solution, and the stability analysis is presented in section 5. In section 6, an application to the waist seal/car window squeal (a rubber/glass contact) is presented. Flutter instabilities with frequencies in the acoustic range, may indeed occur in the studied system. 


\section{THE FRICTIONAL CONTACT PROBLEM IN FINITE ELASTICITY}

\subsection{Finite elasticity for rubberlike materials}

Having in mind the application to a rubberlike waist seal and neglecting the viscosity, an incompressible hyperelastic isotropic material is considered. Let $\mathcal{B}$ be a deformable body occupying the domain $\Omega$ at a reference configuration $\mathcal{C}$. The motion of the body $\mathcal{B}$ is given by the map $\varphi: \bar{\Omega} \times \mathbb{R} \rightarrow \mathbb{R}^{3}, \quad(\mathrm{X}, \tau) \rightarrow \mathrm{X}=\varphi(\mathrm{X}, \tau)=\varphi_{\tau}(\mathrm{X})$. The material constraint of incompressibility is classically written $J=\operatorname{det}(\mathbf{F})=1$, where $\mathbf{F}=\nabla_{X}\left(\boldsymbol{\varphi}_{\tau}\right)$ is the deformation gradient and det denotes the determinant of a second order tensor. In order to overcome locking effects, we choose, among the numerous formulations in the literature ${ }^{5}$, the three-field formulation for quasi-incompressibility proposed by J.C.Simo and R.L.Taylor ${ }^{24}$. This formulation was also used by G.Björkman et al. ${ }^{1}$ for quasistatic frictionless contact problems and is now applied to the present dynamical problem with frictional contact. It is based on the split proposed by P.J.Flory ${ }^{4}$ for the deformation gradient $\mathbf{F}$ and, consequently, for the (right) Cauchy-Green tensor $\mathbf{C}$, into isochoric and volumetric parts :

$$
\mathbf{F}=J^{1 / 3} \tilde{\mathbf{F}} \quad \mathbf{C}=J^{2 / 3} \tilde{\mathbf{C}} \quad \tilde{I}_{3}=1,
$$

where $\tilde{I}_{3}$ is the third principal invariant of $\tilde{\mathbf{C}}$. The volume change is characterized by using a separate field $\theta$, and a third unknown field (a Lagrange multiplier $p$ ) enforces the constraint :

$$
\theta=J
$$

Moreover the volumetric effects on the strain energy and the deviatoric ones are supposed to be uncoupled. This leads to the following expression for the strain energy :

$$
\bar{W}\left(\tilde{I}_{1}, \tilde{I}_{2}, \theta\right)=\tilde{W}\left(\tilde{I}_{1}, \tilde{I}_{2}\right)+U(\theta)
$$

where $\tilde{I}_{1}$ and $\tilde{I}_{2}$ are the first and second principal invariants of $\tilde{\mathbf{C}}$. We have chosen the Mooney Rivlin strain energy that is sufficient for studying the waist seal deformation (the maximum strain numerically obtained for the waist seal is of $50 \%$ ) :

$$
\tilde{W}=a_{1}\left(\tilde{I}_{1}-3\right)+a_{2}\left(\tilde{I}_{2}-3\right)
$$

where $a_{1}$ and $a_{2}$ are the material coefficients. Then the constitutive behavior of the material is characterized by :

$$
\begin{gathered}
\mathbf{S}=p J \mathbf{C}^{-1}+2 J^{-2 / 3} \operatorname{Dev}\left(\frac{\partial \tilde{W}}{\partial \tilde{\mathbf{C}}}\right), \\
p=U^{\prime}(\theta),
\end{gathered}
$$

and $\theta$ satisfies the constraint (2). $\quad \mathbf{S}$ is the second Piola-Kirshoff stress tensor, $\operatorname{Dev}(\mathbf{A})=\mathbf{A}-\frac{1}{3}(\mathbf{A}: \mathbf{C}) \mathbf{C}^{-1}$ and $\partial \tilde{W} / \partial \tilde{\mathbf{C}}=\left(a_{1}+a_{2} \tilde{I}_{1}\right) \mathbb{1}-a_{2} \tilde{\mathbf{C}}$. 


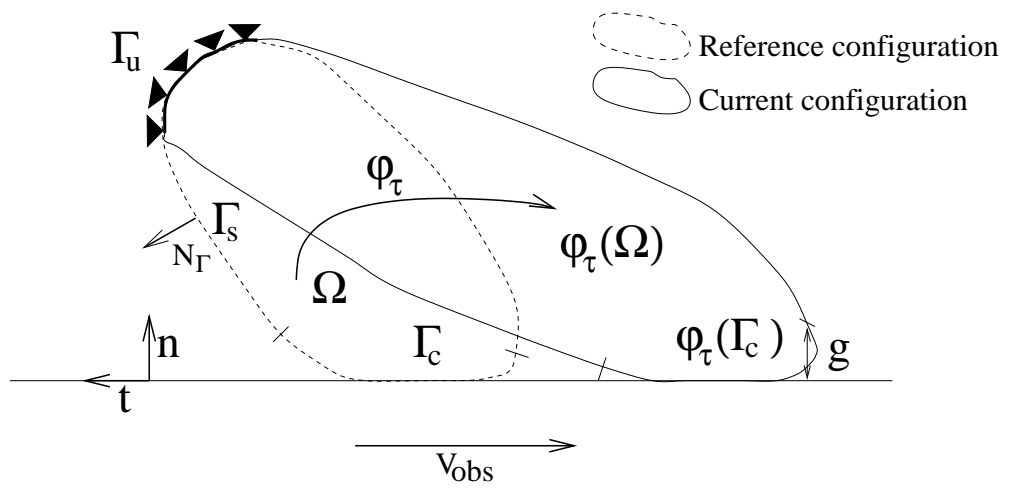

Figure 1: Deformable body in contact with a flat obstacle.

The volumetric part $U$ is similar to a penalty function. It should verify the following properties : $U$ is non-negative over $\mathbb{R}+, U(1)=0$ and $\lim _{J \rightarrow 0} U(\theta)=\infty$. That is the case with the following choice $(\kappa$ is the penalty parameter homogeneous to a bulk modulus) :

$$
U(\theta)=\kappa\left[\frac{1}{2}\left(\theta^{2}-1\right)-\ln (\theta)\right]
$$

\subsection{Frictional contact conditions}

We restrict this study to the two-dimensional problem of the contact between a deformable body and a flat and rigid obstacle that moves with a constant velocity $\mathbf{v}_{o b s}=v_{o b s} \mathbf{t}$ along a direction $\mathbf{t}$ of its surface (see figure 1 ). We denote by $\Gamma_{c}$ the part of the boundary of the reference configuration of the body that contains the particles that may eventually establish contact with the obstacle. The gap between the obstacle surface and the current position $\mathrm{x}$ of such contact candidate particles $\left(\mathrm{x}=\varphi(\mathrm{X}, \tau)\right.$ for $\mathrm{X}$ belonging to $\left.\Gamma_{c}\right)$ is represented by $G(\mathbf{X}, \tau)$ or, equivalently, by $g(\mathbf{x}, \tau)=G\left(\varphi^{-1}(\mathbf{x}, \tau), \tau\right)$. The component along the direction tangent to the obstacle of the velocity of a contact candidate particle relative to the obstacle is represented by $V(\mathbf{X}, \tau)=\dot{\varphi}(\mathbf{X}, \tau) \cdot \mathbf{t}-v_{o b s}$ or, equivalently, by $v(\mathbf{x}, \tau)=V\left(\varphi^{-1}(\mathbf{x}, \tau), \tau\right)$. The contact reactions $\mathbf{r}$ on $\varphi_{\tau}\left(\Gamma_{c}\right)$ are related to the Cauchy stress tensor $\boldsymbol{\sigma}=1 / J \mathbf{F S F} \mathbf{F}^{T}$ by

$$
\mathbf{r}=\boldsymbol{\sigma} \mathbf{n}_{\Gamma},
$$

where $\mathbf{n}_{\Gamma}$ is the normal to the boundary of the body in its current configuration, and the decomposition in normal and tangential components to the obstacle is performed : $\mathbf{r}=$ $r_{n} \mathbf{n}+r_{t} \mathbf{t}, r_{n}=\mathbf{r . n}$ and $r_{t}=$ r.t. The contact is supposed to be unilateral :

$$
g \geq 0, \quad r_{n} \geq 0 \quad \text { and } \quad r_{n} g=0,
$$

and the Coulomb law of friction is adopted :

$$
\left|r_{t}\right|-\mu r_{n} \leq 0, \quad \mu r_{n}|v|=-r_{t} v,
$$

where $\mu$ is the friction coefficient. Unlike most of the classical analyses that associate friction induced instabilities to variations of the coefficient of friction, $\mu$ is taken as a 
$\mathbf{r}(\mathbf{R}) \quad$ Cauchy (nominal) vector of reaction stresses on the contact surface,

$g(G) \quad$ Eulerian (Lagrangean) descriptions of the current gap,

$v_{\text {obs }} \quad$ Absolute value of the tangential velocity of the flat obstacle,

$v(V) \quad$ Eulerian (Lagrangean) descriptions of the current sliding velocity,

$\mathbf{P} \quad$ Vector of dimension $2 N_{c}$ containing the nodal contact reaction forces for the finite element model,

G Vector of dimension $N_{c}$ containing the gaps between the contact candidate nodes and the flat obstacle for the reference configuration of the f.e. mesh,

$\mathbf{H}_{n}\left(\mathbf{H}_{t}\right)$ Transformation matrices of dimension $N_{c} \times N$ that relate the normal (tangential) displacements of the contact candidate nodes of the f.e. mesh to the global vector of nodal displacements.

Table 1: Nomenclature for the main contact variables

constant in the present work. Using the framework of convex analysis ${ }^{7}$, the friction condition (10) becomes :

$$
-r_{t} \in \partial \phi_{r_{n}}(v)
$$

where $\phi_{r_{n}}(y)=\mu r_{n}|y|$ and $\partial \phi_{r_{n}}$ denotes the sub-differential of $\phi_{r_{n}}$. The Coulomb friction condition may also be written as a maximal dissipation principle :

$$
\left|r_{t}\right| \leq \mu r_{n} \quad \text { and } \quad \forall r_{t}^{*} \text { such that }\left|r_{t}^{*}\right| \leq \mu r_{n}, \quad\left(r_{t}^{*}-r_{t}\right) v \geq 0 .
$$

Both contact and friction conditions can be expressed in the reference configuration by using the nominal contact reactions :

$$
\mathbf{R}=\mathbf{F S ~} \mathbf{N}_{\Gamma}
$$

where $\mathbf{N}_{\Gamma}$ is the normal to the boundary $\Gamma$ of the reference configuration of the body, and the decomposition $\mathbf{R}=R_{n} \mathbf{n}+R_{t} \mathbf{t}, R_{n}=\mathbf{R} . \mathbf{n}$ and $R_{t}=\mathbf{R} . \mathbf{t}$ can also be used :

$$
\begin{gathered}
G \geq 0, \quad R_{n} \geq 0 \quad \text { and } \quad R_{n} G=0 \\
\left|R_{t}\right| \leq \mu R_{n} \text { and } \forall R_{t}^{*} \text { such that }\left|R_{t}^{*}\right| \leq \mu R_{n}, \quad\left(R_{t}^{*}-R_{t}\right) V \geq 0 .
\end{gathered}
$$

A nomenclature for the main contact variables can be found in table 1 .

\section{THE DYNAMIC SOLUTION}

\subsection{Formulation and semi-discretization}

In this subsection, we are interested in the computation of the dynamic behavior of the solid $\mathcal{B}$. The perfect unilateral contact and Coulomb friction conditions induce mathematical difficulties that are still unresolved for continuum bodies. Thus the equations of 
elastodynamics and the corresponding variational problem are formally stated for sufficiently regular solutions. After space discretization we will state a problem that takes into account discontinuities of the contact velocity ${ }^{7}$. At any instant $\tau$, the equation of linear momentum balance is :

$$
\rho \ddot{\varphi}-\operatorname{Div}(\mathbf{F S})=\mathbf{f}_{v},
$$

where $\rho$ is the density of the body in its reference configuration and $\mathbf{f}_{v}$ is the external force that acts on $\Omega$. The boundary conditions are given by the prescribed configuration on $\Gamma_{u}$

$$
\varphi_{\tau}=\varphi_{i m p} \text { on } \Gamma_{u}
$$

by the surface tractions $\mathbf{f}_{s}$ on $\Gamma_{s}$,

$$
(\mathbf{F S}) \mathbf{N}_{\Gamma}=\mathbf{f}_{s},
$$

where $\mathbf{N}_{\Gamma}$ is the outward unit normal vector to $\Gamma$, and by the frictional contact conditions on $\Gamma_{c}\left(\Gamma=\bar{\Gamma}_{u} \cup \bar{\Gamma}_{s} \cup \bar{\Gamma}_{c}\right.$ and $\Gamma_{u}, \Gamma_{s}$ and $\Gamma_{c}$ being disjoint $)$. The initial conditions are given by the initial positions and the initial velocities.

\subsubsection{Weak formulation}

Considering the specific form (7) of the volumetric part $U$ of the strain energy $\bar{W}$, we suppose that no violation of the kinematic constraint $\operatorname{det}\left(\nabla_{\boldsymbol{X}}\left(\boldsymbol{\varphi}_{\tau}\right)\right)>0$ is possible ${ }^{6}$. The set of admissible configurations is simply :

$$
\mathcal{W}=\left\{\varphi_{\tau}: \Omega \longrightarrow \mathbb{R}^{2} \text { such that } \varphi_{\tau}(\mathbf{X})=\varphi_{i m p} \quad \forall \mathbf{X} \in \Gamma_{u}\right\}
$$

The set of admissible variations to the reference configuration is :

$$
T^{0} \mathcal{W}=\left\{\boldsymbol{\eta}: \Omega \longrightarrow \mathbb{R}^{2} \text { such that } \boldsymbol{\eta}(\mathbf{X})=0 \forall \mathbf{X} \in \Gamma_{u}\right\}
$$

The set of admissible hydrostatic pressures and volume changes is :

$$
\mathcal{V}=\left\{\theta^{*}: \Omega \longrightarrow \mathbb{R}\right\}
$$

Finally, the set of admissible normal contact stresses is :

$$
C_{n}=\left\{R_{n}^{*}: \Gamma_{c} \longrightarrow \mathbb{R} \text { such that } R_{n}^{*} \geq 0\right\} .
$$

Except for the inertia forces, all the non-dissipative effects can be put together in the functional $\Pi$ proposed by G.Björkmann et al. ${ }^{1}$ :

$$
\Pi\left(\varphi_{\tau}, \theta, p, R_{n}\right)=\Pi_{i n t}\left(\varphi_{\tau}, \theta, p\right)-\Pi_{e x t}\left(\varphi_{\tau}\right)-\int_{\Gamma_{c}} R_{n} G d \Gamma
$$

with $\left(\varphi_{\tau}, \theta, p, R_{n}\right) \in \mathcal{W} \times \mathcal{V} \times \mathcal{V} \times C_{n} ; \Pi_{\text {int }}$ denotes an augmented potential of the internal forces :

$$
\Pi_{i n t}\left(\boldsymbol{\varphi}_{\tau}, \theta, p\right)=\int_{\Omega}\left[\tilde{W}\left(\tilde{I}_{1}, \tilde{I}_{2}\right)+U(\theta)+p(J-\theta)\right] d V
$$


and $\Pi_{e x t}$ denotes the potential of the external forces :

$$
\Pi_{e x t}\left(\boldsymbol{\varphi}_{\tau}\right)=\int_{\Omega} \mathbf{f}_{v} \boldsymbol{\varphi}_{\tau} d V+\int_{\Gamma_{s}} \mathbf{f}_{s} \boldsymbol{\varphi}_{\tau} d \Gamma
$$

The following pseudo-potential represents the dissipation due to friction :

$$
D\left(R_{n}, \dot{\varphi_{\tau}}-\dot{\Phi}\right)=\int_{\Gamma_{c}} \mu R_{n}\left|\left(\dot{\varphi}_{\tau}-\dot{\Phi}\right) \cdot \mathbf{t}\right| d \Gamma
$$

where $\dot{\boldsymbol{\Phi}}$ is an application that verifies $\dot{\boldsymbol{\Phi}} \in \mathcal{W}$ and $\dot{\boldsymbol{\Phi}}(\mathbf{X})=\mathbf{v}_{\text {obs }}$ on $\Gamma_{c}$. Taking into account the Coulomb friction condition (11) and using the notion of sub-differential of a convex function (see ${ }^{23}$ ), the variational problem can be written as an inclusion ${ }^{7}$ :

$$
-\Pi_{, \varphi}\left(\boldsymbol{\varphi}_{\tau}, \theta, p, R_{n}\right)-\mathcal{F}_{\text {ine }} \in \partial_{2} D\left(R_{n}, \dot{\varphi_{\tau}}-\dot{\boldsymbol{\Phi}}\right),
$$

together with the following variational equations and inequality :

$$
\begin{gathered}
\forall \theta^{*} \in \mathcal{V}, \quad \Pi_{i n t, \theta} \theta^{*}=\int_{\Omega}\left(U^{\prime}(\theta)-p\right) \theta^{*} d V=0, \\
\forall p^{*} \in \mathcal{V}, \quad \Pi_{i n t, p} p^{*}=\int_{\Omega}(J-\theta) p^{*} d V=0, \\
\forall R_{n}^{*} \in C_{n}, \quad \int_{\Gamma_{c}}\left(R_{n}^{*}-R_{n}\right) G d \Gamma \geq 0,
\end{gathered}
$$

where $\mathcal{F}_{\text {ine }}$ denotes the inertia forces and $\partial_{2} D$ the sub-differential of $D$ with respect to its second argument. Using the definition of the sub-differential of a convex function, the inclusion (27) may be replaced by the variational inequality :

$$
\begin{aligned}
& \forall \varphi^{*} \in \mathcal{W}, \\
& -<\Pi_{, \varphi}+\mathcal{F}_{i n e} ; \varphi^{*}-\dot{\varphi}_{\tau}>\leq D\left(R_{n}, \varphi^{*}-\dot{\Phi}\right)-D\left(R_{n}, \dot{\varphi}_{\tau}-\dot{\Phi}\right) .
\end{aligned}
$$

If the Coulomb friction condition is expressed in terms of the maximal dissipation principle (12) and tangential nominal contact reactions $R_{t}$ are considered as an additional unknown field, then the variational inequality (31) is replaced by the following variational equation (d'Alembert's principle) :

$$
\forall \boldsymbol{\eta} \in \mathcal{W}, \quad\left(\Pi_{i n t, \varphi}-\Pi_{e x t, \varphi}+\mathcal{F}_{i n e}\right) \cdot \boldsymbol{\eta}-\int_{\Gamma_{c}}\left(R_{n} \mathbf{n}+R_{t} \mathbf{t}\right) \cdot \boldsymbol{\eta} d \Gamma=0,
$$

together with the variational inequality (Coulomb friction condition) :

$$
\forall R_{t}^{*} \in C_{t}\left(R_{n}\right), \quad \int_{\Gamma_{c}}\left(R_{t}^{*}-R_{t}\right) V d \Gamma \geq 0
$$

with

$$
\Pi_{i n t, \varphi} \cdot \boldsymbol{\eta}=\int_{\Omega} \mathbf{F S}:\left(\frac{\partial \boldsymbol{\eta}}{\partial \mathbf{X}}\right) d V
$$




$$
\Pi_{e x t, \varphi} \cdot \boldsymbol{\eta}=\int_{\Omega} \mathbf{f}_{v} \boldsymbol{\eta} d V+\int_{\Gamma_{s}} \mathbf{f}_{s} \boldsymbol{\eta} d \Gamma
$$

and where $C_{t}\left(R_{n}\right)$ is the set of admissible tangential contact reactions :

$$
C_{t}\left(R_{n}\right)=\left\{R_{t}^{*}: \Gamma_{c} \longrightarrow \mathbb{R} \text { such that }\left|R_{t}^{*}\right| \leq \mu R_{n}\right\} .
$$

Summarizing, the dynamical frictional contact problem P1 is governed at each time $\tau$ by the set of variational equations and inequalities (28)-(31) involving the unknown fields $\varphi, \theta, p$ and $R_{n}$ or, equivalently, by the variational statements (28)-(30), (32) and (33) involving the unknown fields $\varphi, \theta, p, R_{n}$ and $R_{t}$.

\subsubsection{Space discretization}

Many details about the space dicretization associated to the three-field formulation may be found in $1,12,24$. The number of unknowns may be reduced by eliminating the two auxilary fields $\theta$ and $p$ at the element level. Indeed the most important feature is that the shape functions of $\theta$ and $p$ are chosen to be discontinuous over the element boundaries. That is the case of the finite element Q4P0 used in this study (four nodes for the displacement interpolation and one node for the interpolation of the volume change and the hydrostatic pressure). If $\theta_{e}^{h}$ and $p_{e}^{h}$ denote the discretized forms of the volume change and of the pressure on some finite element $\Omega_{e}^{h}$, the equations (28) and (29) yield the following relations :

$$
\theta_{e}^{h}=\frac{\operatorname{Vol}\left(\boldsymbol{\varphi}_{\tau}\left(\Omega_{e}^{h}\right)\right)}{\operatorname{Vol}\left(\Omega_{e}^{h}\right)}, \quad \quad p_{e}^{h}=U^{\prime}\left(\theta_{e}^{h}\right)
$$

Then, at the element level, $\theta_{e}^{h}$ and $p_{e}^{h}$ are replaced in the equations of motion by the relations $(37)^{24}$.

The integrals over the boundary $\Gamma$ are discretized by using the trapezoidal rule. The integration points are the nodes of the displacement interpolation. We denote by $\mathbf{P}_{n}$ the vector (of dimension $N_{c}$ ) of normal nodal contact forces (respectively $\mathbf{P}_{t}$ for the tangential nodal contact forces) where $N_{c}$ is the number of contact candidate nodes. The vector $\mathbf{P}$ of dimension $2 * N_{c}$ puts together the components of $\mathbf{P}_{n}$ and $\mathbf{P}_{t}$. We denote by $\mathrm{U}$ the vector of dimension $N$ of nodal displacements ( $N$ is the total number of degrees of freedom). The normal and tangential components of the displacement of the contact node number $i$ are given by :

$$
U_{n_{i}}=\mathbf{H}_{n_{i}} \mathbf{U} \quad U_{t_{i}}=\mathbf{H}_{t_{i}} \mathbf{U}
$$

where $\mathbf{H}_{n_{i}}$ and $\mathbf{H}_{t_{i}}$ are two vectors of dimension $N$. The matrix $\mathbf{H}_{n}$ (respectively $\mathbf{H}_{t}$ ) of dimension $N_{c} \times N$ puts together the $N_{c}$ vectors $\mathbf{H}_{n_{i}}$ (respectively $\mathbf{H}_{t_{i}}$ ). We denote also by $\mathbf{H}$ the matrix of dimension $2 * N_{c} \times N$ such that $\mathbf{H}^{T}=\left[\mathbf{H}_{n}^{T}, \mathbf{H}_{t}^{T}\right]$. The sets $C_{n}$ and $C_{t}\left(R_{n}\right)$ are discretized in $C_{n}^{h}$ and $C_{t}^{h}\left(\mathbf{P}_{n}\right)$ :

$$
\begin{aligned}
& C_{n}^{h}=\left\{\mathbf{P}_{n} \in \mathbb{R}^{N_{c}} \text { such that } P_{n_{i}} \geq 0 \forall i=1, . ., N_{c}\right\}, \\
& C_{t}^{h}\left(\mathbf{P}_{n}\right)=\left\{\mathbf{P}_{t} \in \mathbb{R}^{N_{c}} \text { such that }\left|P_{t_{i}}\right| \leq \mu P_{n_{i}} \forall i=1, . ., N_{c}\right\} .
\end{aligned}
$$


Using the previous notations, the discretized form of the variational inequality (30) of the unilateral contact condition is :

$$
\forall \mathbf{P}_{n}^{*} \in C_{n}^{h} \quad\left(\mathbf{P}_{n}^{*}-\mathbf{P}_{n}\right)^{T}\left(\mathbf{H}_{n} \mathbf{U}+\mathbf{G}\right) \geq 0
$$

where each component of the vector $\mathbf{G}$ (of dimension $N_{c}$ ) is equal to the corresponding reference gap. The discretized form of the variational inequality (33) of the friction conditions is :

$$
\forall \mathbf{P}_{t}^{*} \in C_{t}^{h}\left(\mathbf{P}_{n}\right) \quad\left(\mathbf{P}_{t}^{*}-\mathbf{P}_{t}\right)^{T}\left(\mathbf{H}_{t} \dot{\mathbf{U}}-\mathbf{V}_{o b s}\right) \geq 0,
$$

where the components of the vector $\mathbf{V}_{o b s}$ (of dimension $N_{c}$ ) are equal to $v_{o b s}$. The semidiscrete equation of motion is :

$$
\mathbf{M U}+\mathbf{F}_{\text {int }}(\mathbf{U})-\mathbf{F}_{\text {ext }}=\mathbf{H}^{T} \mathbf{P}
$$

where $\mathbf{M}, \mathbf{F}_{\text {int }}$ and $\mathbf{F}_{\text {ext }}$ denote the consistent mass matrix, the internal forces and the external forces, respectively. The semi-discrete Problem P2 is thus given by the equation of motion (42), the initial displacements $\mathbf{U}^{0}$ and the initial velocities $\dot{\mathbf{U}}^{0}$ together with the frictional contact conditions (40) and (41).

Finite dimensional dynamical contact problems are not regular because of the discontinuities of the gap velocity when impact occurs. In ${ }^{7,8}$ the problem is set so as to take into account those discontinuities. Following J.J.Moreau ${ }^{7}$, we make the assumption that the velocity has a variation bounded in time so as to define at any instant a left $\dot{\mathbf{U}}^{-}$ and a right $\dot{\mathbf{U}}^{+}$velocity. We suppose also that the velocity is right-continuous and we denote by $\dot{\mathbf{U}}=\dot{\mathbf{U}}^{+}$the velocity at any instant. The acceleration should be understood as a differential measure $d \dot{\mathbf{U}}$ :

$$
\int_{] a, b]} d \dot{\mathbf{U}}=\dot{\mathbf{U}}^{+}(b)-\dot{\mathbf{U}}^{+}(a)
$$

and the equation of motion (42) as a measure differential equation :

$$
\mathbf{M} d \dot{\mathbf{U}}+\mathbf{F}_{\text {int }}(\mathbf{U}) d \tau-\mathbf{F}_{\text {ext }} d \tau-\mathbf{H}^{T} \mathbf{P} d \nu=0
$$

where $d \nu$ is some non-negative measure relative to which $d \dot{\mathbf{U}}$ possesses a density function and $\mathbf{P}$ is the representative of the local density of impulses exerted when contact occurs.

\subsection{Approximation in time}

Following S.Pandit and S.Deo ${ }^{18}$, one obtains :

$$
\left\{\begin{array}{l}
\mathbf{M}(\dot{\mathbf{U}}(\tau)-\dot{\mathbf{U}}(0))=\int_{0}^{\tau}\left(\mathbf{F}_{\text {ext }}-\mathbf{F}_{\text {int }}(\mathbf{U})\right) d \tau+\int_{] 0, \tau]} \mathbf{H}^{T} \mathbf{P} d \nu \\
\mathbf{U}(\tau)-\mathbf{U}(0)=\int_{0}^{\tau} \dot{\mathbf{U}} d \tau
\end{array}\right.
$$


After dividing $[0, T]$ into sub-intervals, several time approximations of (44) may be used, see ${ }^{25}$. We have chosen the following one :

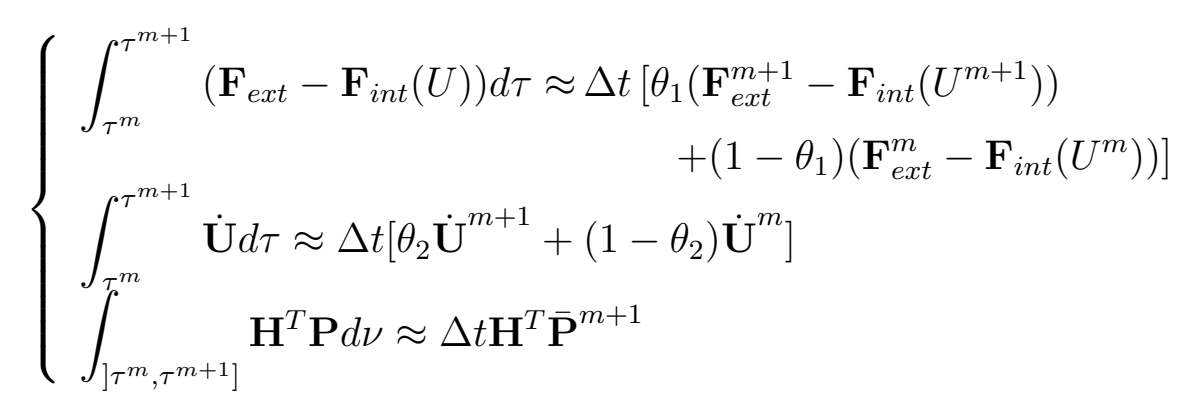

where $\square^{m}$ denotes the approximation of $\square\left(\tau^{m}\right), \Delta t=\tau^{m+1}-\tau^{m}, \theta_{1}$ and $\theta_{2}$ are two numerical coefficients in the interval $[0,1]$ and $\overline{\mathbf{P}}^{m+1}$ is the mean value of the impulse density in the time interval.

Using the second approximation of (46), the contact condition is written with the velocity as unknown :

$$
\left\{\begin{array}{l}
\overline{\mathbf{P}}_{n}^{m+1} \in C_{n}^{h} \text { and } \\
\forall \mathbf{P}_{n}^{*} \in C_{n}^{h}\left(\mathbf{P}_{n}^{*}-\overline{\mathbf{P}}_{n}^{m+1}\right)^{T}\left(\mathbf{H}_{n} \dot{\mathbf{U}}^{m+1}+\tilde{\mathbf{G}}^{m}\right) \geq 0 \\
\tilde{\mathbf{G}}^{m^{m}}=\left(1-\frac{1}{\theta_{2}}\right) \mathbf{H}_{n} \dot{\mathbf{U}}^{m}+\frac{1}{\Delta t \theta_{2}}\left(\mathbf{H}_{n} \mathbf{U}^{m}+\mathbf{G}\right)
\end{array}\right.
$$

The discretized Coulomb condition involves the tangential relative velocity $\mathbf{H}_{t} \dot{\mathbf{U}}^{m+1}$ $\mathbf{V}_{\text {obs }}$ and the average contact force $\overline{\mathbf{P}}^{m+1}$. Finally the discrete non-linear problem is the following one :

\section{Problem P3:}

find $\dot{\mathbf{U}}^{m+1} \in \mathbb{R}^{N}, \overline{\mathbf{P}}_{n}^{m+1} \in C_{n}^{h}$ and $\overline{\mathbf{P}}_{t}^{m+1} \in C_{t}^{h}\left(\overline{\mathbf{P}}_{n}^{m+1}\right)$ such that :

$$
\left\{\begin{array}{l}
\mathbf{M}^{m+1}+\Delta t \theta_{1}\left(\mathbf{F}_{\text {int }}\left(l\left(\dot{\mathbf{U}}^{m+1}\right)\right)-\mathbf{F}_{e x t}^{m+1}\right)=\Delta t \mathbf{F}_{r e s}^{m}+\Delta t \mathbf{H}^{T} \overline{\mathbf{P}}^{m+1} \\
\forall \mathbf{P}_{n}^{*} \in C_{n}^{h} \quad\left(\mathbf{P}_{n}^{*}-\overline{\mathbf{P}}_{n}^{m+1}\right)^{T}\left(\mathbf{H}_{n} \dot{\mathbf{U}}^{m+1}+\tilde{\mathbf{G}}^{m}\right) \geq 0 \\
\forall \mathbf{P}_{t}^{*} \in C_{t}^{h}\left(\overline{\mathbf{P}}_{n}^{m+1}\right) \quad\left(\mathbf{P}_{t}^{*}-\overline{\mathbf{P}}_{t}^{m+1}\right)^{T}\left(\mathbf{H}_{t} \dot{\mathbf{U}}^{m+1}-\mathbf{V}_{o b s}\right) \geq 0
\end{array}\right.
$$

with

$$
\begin{aligned}
& l\left(\dot{\mathbf{U}}^{m+1}\right)=\frac{1}{\Delta \theta_{2}}\left(\mathbf{U}^{m+1}-\mathbf{U}^{m}\right)-\frac{1-\theta_{2}}{\theta_{2}} \dot{\mathbf{U}}^{m} \\
& \mathbf{F}_{\text {res }}^{m}=\frac{1}{\Delta t} \mathbf{M U}^{m}+\left(1-\theta_{1}\right)\left[\mathbf{F}_{\text {ext }}^{m}-\mathbf{F}_{\text {int }}\left(l\left(\dot{\mathbf{U}}^{m}\right)\right)\right] .
\end{aligned}
$$

\subsection{Resolution}

In order to linearize the problem (48) which is constrained by variational inequalities, it is formulated in terms of generalized equations. The variational inequality ( $C$ is a convex set) :

$$
\mathbf{z} \in C \text { and } \forall \mathbf{z}^{*} \in C \quad \mathcal{F}(\mathbf{z})\left(\mathbf{z}^{*}-\mathbf{z}\right) \geq 0
$$


is equivalent to the generalized equation :

$$
-\mathcal{F}(\mathbf{z}) \in \partial \psi_{C}(\mathbf{z})
$$

where $\psi_{C}$ is the indicator function of the convex set $C$. Following A.Klarbring and G.Björkmann ${ }^{11}$, we suppose that implicit sets such as $C_{t}^{h}\left(\overline{\mathbf{P}}_{n}^{m+1}\right)$ may also be considered. For simplicity, the superscript ${ }^{m+1}$ is now omitted. In the case of the dynamical problem, we consider : $C=\mathbb{R}^{N} \times C_{n}^{h} \times C_{t}^{h}\left(\overline{\mathbf{P}}_{n}\right)$ and

$$
\mathbf{z}=\left[\begin{array}{c}
\dot{\mathbf{U}} \\
\overline{\mathbf{P}}_{n} \\
\overline{\mathbf{P}}_{t}
\end{array}\right], \quad \mathcal{F}(\mathbf{z})=\left[\begin{array}{c}
\mathcal{L}\left(\dot{\mathbf{U}}, \overline{\mathbf{P}}_{n}, \overline{\mathbf{P}}_{t}\right) \\
\tilde{\mathbf{G}}_{n}^{m}+\mathbf{H}_{n} \dot{\mathbf{U}} \\
\mathbf{V}_{o b s}-\mathbf{H}_{t} \dot{\mathbf{U}}
\end{array}\right]
$$

with $\mathcal{L}\left(\dot{\mathbf{U}}, \overline{\mathbf{P}}_{n}, \overline{\mathbf{P}}_{t}\right)=\mathbf{F}_{r e s}^{m}+\mathbf{H}_{n}^{T} \overline{\mathbf{P}}_{n}+\mathbf{H}_{t}^{T} \overline{\mathbf{P}}_{t}-\frac{1}{\Delta t} \mathbf{M U}^{m}-\theta_{1} \mathbf{F}_{\text {int }}(l(\dot{\mathbf{U}}))$.

The Newton-Raphson algorithm may be applied to the generalized equations ${ }^{3}$. Considering the properties of $\mathbf{H}$, the linearisation leads to the following problem.

Problem P4: Assuming that the evolution is known until the instant $\tau^{m}$ and that $\left(\dot{\mathbf{U}}_{0},\left(\overline{\mathbf{P}}_{n}\right)_{0},\left(\overline{\mathbf{P}}_{t}\right)_{0}\right), . .,\left(\dot{\mathbf{U}}_{k},\left(\overline{\mathbf{P}}_{n}\right)_{k},\left(\overline{\mathbf{P}}_{t}\right)_{k}\right)$ are known, find $\left(\dot{\mathbf{U}}_{k+1},\left(\overline{\mathbf{P}}_{n}\right)_{k+1},\left(\overline{\mathbf{P}}_{t}\right)_{k+1}\right) \in \mathbb{R}^{N} \times C_{n}^{h} \times C_{t}^{h}\left(\left(\overline{\mathbf{P}}_{n}\right)_{k+1}\right)$ such that :

$$
\left\{\begin{array}{l}
\hat{\mathbf{K}}_{k} d \dot{\mathbf{U}}_{k+1}=\hat{\mathbf{F}}_{k}+\mathbf{H}_{n}^{T}\left(\overline{\mathbf{P}}_{k+1}\right)_{n}+\mathbf{H}_{t}^{T}\left(\overline{\mathbf{P}}_{k+1}\right)_{t} \\
\overline{\mathbf{P}}_{n_{k+1}} \in C_{n}^{h} \text { and } \forall \mathbf{P}_{n}^{*} \in C_{n}^{h} \\
\left(\mathbf{P}_{n}^{*}-\left(\overline{\mathbf{P}}_{n}\right)_{k+1}\right)^{T}\left(\tilde{\mathbf{G}}_{n}^{m}+\mathbf{H}_{n} \dot{\mathbf{U}}_{k}+\mathbf{H}_{n} d \dot{\mathbf{U}}_{k+1}\right) \geq 0 \\
\left(\overline{\mathbf{P}}_{t}\right)_{k+1} \in C_{t}^{h}\left(\left(\overline{\mathbf{P}}_{n}\right)_{k+1}\right) \text { and } \forall \mathbf{P}_{t}^{*} \in C_{t}^{h}\left(\left(\overline{\mathbf{P}}_{n}\right)_{k+1}\right) \\
\left(\mathbf{P}_{t}^{*}-\left(\overline{\mathbf{P}}_{t}\right)_{k+1}\right)^{T}\left(\mathbf{H}_{t} \dot{\mathbf{U}}_{k}+\mathbf{H}_{t} d \dot{\mathbf{U}}_{k+1}-\mathbf{V}_{o b s}\right) \geq 0
\end{array}\right.
$$

with $\hat{\mathbf{F}}_{k}=\theta_{1} \mathbf{F}_{\text {ext }}+\mathbf{F}_{\text {Res }}^{m}-\frac{1}{\Delta t} \mathbf{M} \dot{\mathbf{U}}_{k+1}-\theta_{1} \mathbf{F}_{\text {int }}\left(l\left(\dot{\mathbf{U}}_{k}\right)\right)$ and $d \dot{\mathbf{U}}_{k+1}=\dot{\mathbf{U}}_{k+1}-\dot{\mathbf{U}}_{k} . \hat{\mathbf{K}}_{k}$ is the matrix of iterations : $\hat{\mathbf{K}}_{k}=\frac{1}{\Delta t} \mathbf{M}+\Delta t \theta_{1} \theta_{2} \mathbf{K}_{k}$ where $\mathbf{K}_{k}=\partial \mathbf{F}_{\text {int }} / \partial \mathbf{U}$ is the tangent stiffness matrix which is described in details in ${ }^{12}$.

The problem (53) is then formulated in terms of complementarity and, after a condensation on the contact node variables, it is solved by using the Lemke method. This process has been detailled by A.Klarbring and G.Björkmann ${ }^{11}$ and by P.Chabrand et al. ${ }^{2}$ for a quasistatic problem. The Lemke mathematical programming method is a direct pivoting algorithm which turns out to be very fast ${ }^{3}$.

\section{THE STEADY SLIDING SOLUTION}

In the present case, as for disk brakes ${ }^{16}$, we are interested in instabilities that may occur when a steady frictional regime is desired. Therefore, we propose an algorithm to determine directly that steady sliding solution. The steady sliding problem was mathematically studied for a linear elastic body by P.Rabier et al. ${ }^{20}$ (normal compliance law) and by E.B.Pires et al. ${ }^{19}$ (non-local friction law). Finite dimensional coercive and 
non-coercive versions of the steady sliding problem with classical unilateral contact conditions were studied by A.Klarbring ${ }^{9,10}$, .

In this section a method is proposed to find a steady sliding equilibrium configuration of a non-linear elastic solid in contact with a flat obstacle that has a constant tangential velocity $v_{o b s}$. The relative tangential velocity of the nodes in contact is equal to $-v_{o b s}$ and, consequently, the classical Coulomb friction law simplifies to :

$$
r_{t} v_{o b s}=\mu\left|v_{o b s}\right| r_{n}
$$

For this particular case of frictional contact problems, the direction of the tangential contact stress is known. Thus the problem becomes simpler because the non-smooth character of the friction law is eliminated. We just get the extra relation (54) between the normal and the tangential components of the contact stresses. A new problem is derived from the problem P3, see (48) :

$$
\begin{aligned}
& \underline{\text { Problem P5 }} \text { : find }\left(\mathbf{U}, \mathbf{P}_{n}\right) \in \mathbb{R}^{N} \times C_{n}^{h} \text { such that : } \\
& \left\{\begin{array}{l}
\mathbf{F}_{\text {int }}(\mathbf{U})=\mathbf{F}_{\text {ext }}+\mathbf{H}_{n} \mathbf{P}_{n}+\mu \operatorname{sgn}\left(v_{\text {obs }}\right) \mathbf{H}_{t} \mathbf{P}_{n} \\
\forall \mathbf{P}_{n}^{*} \in C_{n}^{h} \quad\left(\mathbf{P}_{n}^{*}-\mathbf{P}_{n}\right)^{T}\left(\mathbf{H}_{n} \mathbf{U}+\mathbf{G}\right) \geq 0
\end{array}\right.
\end{aligned}
$$

where $\operatorname{sgn}(x)=x /|x|$ if $x \neq 0$. One obtains a sequence of frictionless contact problems by using a fixed point procedure on the frictional tangential contact forces. At each fixed point iteration, the tangential contact forces are updated by using the normal contact forces computed previously and the corresponding problem is :

Problem P6 : $\left(\mathbf{U}^{0}, \mathbf{P}_{n}^{0}\right), . .,\left(\mathbf{U}^{l-1}, \mathbf{P}_{n}^{l-1}\right)$ being known, find $\left(\mathbf{U}^{l}, \mathbf{P}_{n}^{l}\right) \in \mathbb{R}^{N} \times C_{n}^{h}$ such that :

$$
\left\{\begin{array}{l}
\mathbf{F}_{\text {int }}\left(\mathbf{U}^{l}\right)^{n}=\mathbf{F}_{\text {ext }}+\mathbf{H}_{n}^{T} \mathbf{P}_{n}^{l}+\mu \operatorname{sgn}\left(v_{o b s}\right) \mathbf{H}_{t}^{T} \mathbf{P}_{n}^{l-1} \\
\forall \mathbf{P}_{n}^{*} \in C_{n}^{h} \quad\left(\mathbf{P}_{n}^{*}-\mathbf{P}_{n}^{l}\right)^{T}\left(\mathbf{H}_{n} \mathbf{U}^{l}+\mathbf{G}\right) \geq 0
\end{array}\right.
$$

where $\square^{l}$ denotes the value of $\square$ obtained at the fixed point iteration $l$. This problem is then linearized similarly to (48). At each iteration of the Newton-Raphson algorithm, the following problem is solved :

Problem P7 : $\left(\mathbf{U}_{0}^{l},\left(\mathbf{P}_{n}^{l}\right)_{0}\right), \ldots,\left(\mathbf{U}_{k}^{l},\left(\mathbf{P}_{n}^{l}\right)_{k}\right)$ being known, find $\left(\mathbf{U}_{k+1}^{l},\left(\mathbf{P}_{n}^{l}\right)_{k+1}\right) \in \mathbb{R}^{N} \times C_{n}^{h}$ such that :

$$
\left\{\begin{array}{l}
\mathbf{K}_{k} d \mathbf{U}_{k+1}^{l}=\mathbf{F}_{e x t}-\mathbf{F}_{i n t}\left(\mathbf{U}_{k}^{l}\right)+\mathbf{H}_{n}^{T}\left(\mathbf{P}_{n}^{l}\right)_{k+1}+\mu \operatorname{sgn}\left(v_{o b s}\right) \mathbf{H}_{t}^{T} \mathbf{P}_{n}^{l-1} \\
\forall \mathbf{P}_{n}^{*} \in C_{n}^{h} \quad\left(\mathbf{P}_{n}^{*}-\left(\mathbf{P}_{n}^{l}\right)_{k+1}\right)\left(\mathbf{H}_{n} \mathbf{U}_{k}^{l}+\mathbf{H}_{n} d \mathbf{U}_{k+1}^{l}+\mathbf{G}\right) \geq 0
\end{array}\right.
$$

where $\square_{k}^{l}$ denotes the value of $\square$ obtained at the fixed point iteration $l$ and at the Newton-Raphson iteration $k$ and where $d \mathbf{U}_{k+1}^{l}=\mathbf{U}_{k+1}^{l}-\mathbf{U}_{k}^{l}$. The problem P7 (see (57)) is also formulated in terms of complementarity and solved by using the Lemke method.

The initial condition for the fixed point procedure is a steady sliding equilibrium position obtained with $\mu=0$ or with a smaller value of $\mu$. 
The advantage of this method is to get the solutions for various friction coefficients. This is very interesting because the friction coefficient is a key parameter in the occurrence of instabilities. An analysis of the sensitivity of the phenomenon relatively to this parameter will be possible without extra computations.

\section{INSTABILITY OF STEADY SLIDING STATES}

In this section we are interested in the occurrence of dynamic instabilities of steady sliding equilibrium states of finite dimensional systems. In other words, we will study the possible existence of growing dynamic solutions in the neighborhood of those equilibrium states. We will show in section 6 that, in the present case, oscillatory (flutter) instabilities are found.

For any given non-vanishing velocity of the flat surface, the sliding velocity of all the nodes in contact at a steady sliding equilibrium configuration is different from zero. We assume, in addition, that the normal reactions of all those contact nodes are strictly positive at the steady sliding state. Thus each contact node is either in (strict) sliding contact $\left(P_{n_{i}}^{0}>0, U_{n_{i}}^{0}+G_{i}=0, P_{t_{i}}^{0}=\mu \operatorname{sgn}\left(v_{o b s}\right) P_{n_{i}}^{0}\right.$ and $\left.\dot{U}_{t_{i}}^{0}=0\right)$ or in (strict) separate contact $\left(P_{t_{i}}^{0}=P_{n_{i}}^{0}=0\right.$ and $\left.U_{n_{i}}^{0}+G_{i}>0\right)$ where $\square^{0}$ denotes the value of $\square$ at the steady sliding equilibrium configuration. Then we consider perturbed dynamic solutions in a neighborhood of this steady sliding state, such that the contact state of each contact candidate node is preserved : each node in sliding contact remains in sliding contact $\left(U_{n_{i}}+G_{i}=\dot{U}_{n_{i}}=0, P_{t_{i}}=\mu \operatorname{sgn}\left(P_{t_{i}}^{0}\right) P_{n_{i}}\right.$ and $\left.\dot{U}_{t_{i}} \neq v_{o b s}\right)$, and each node not in contact remains so with $\left(P_{t_{i}}=P_{n_{i}}=0\right.$ and $\left.U_{n_{i}}+G_{i}>0\right)$. Using the subscript $f$ (free) for the degrees of freedom that correspond to displacement components of node not candidate to contact or not currently in contact, and the subscript $n$ (respectively $t$ ) for those that correspond to normal (respectively tangential) displacements of sliding nodes, the dynamical problem may be written :

$$
\left[\begin{array}{lll}
\mathbf{M}_{f f} & \mathbf{M}_{f n} & \mathbf{M}_{f t} \\
\mathbf{M}_{n f} & \mathbf{M}_{n n} & \mathbf{M}_{n t} \\
\mathbf{M}_{t f} & \mathbf{M}_{t n} & \mathbf{M}_{t t}
\end{array}\right]\left[\begin{array}{l}
\ddot{\mathbf{U}}_{f} \\
0 \\
\ddot{\mathbf{U}}_{t}
\end{array}\right]+\left[\begin{array}{l}
\mathbf{F}_{i n t_{f}}\left(\mathbf{U}_{f}, \mathbf{U}_{t}\right) \\
\mathbf{F}_{i n t_{n}}\left(\mathbf{U}_{f}, \mathbf{U}_{t}\right) \\
\mathbf{F}_{i n t_{t}}\left(\mathbf{U}_{f}, \mathbf{U}_{t}\right)
\end{array}\right]=\left[\begin{array}{l}
0 \\
\mathbf{P}_{n} \\
\mu s \mathbf{P}_{n}
\end{array}\right]
$$

where $s=\operatorname{sgn}\left(v_{o b s}\right)$. Using the equations of motion corresponding to the normal degrees of freedom of the sliding nodes $(n)$ to eliminate the non vanishing reactions, the equations that govern the smooth dynamic evolution of the structure in that neighborhood are :

$$
\underbrace{\left[\begin{array}{ll}
\mathbf{M}_{f f} & \mathbf{M}_{f t} \\
\mathbf{M}_{t f}^{*} & \mathbf{M}_{t t}^{*}
\end{array}\right]}_{\mathbf{M}^{*}}\left[\begin{array}{c}
\ddot{\mathbf{U}}_{f} \\
\ddot{\mathbf{U}}_{t}
\end{array}\right]+\left[\begin{array}{l}
\mathbf{F}_{\text {int }}\left(\mathbf{U}_{f}, \mathbf{U}_{t}\right) \\
\mathbf{F}_{\text {int }_{t}}^{*}\left(\mathbf{U}_{f}, \mathbf{U}_{t}\right)
\end{array}\right]=\left[\begin{array}{l}
0 \\
0
\end{array}\right]
$$

where $\mathbf{F}_{\text {int } t_{t}}^{*}=\mathbf{F}_{\text {int }_{t}}-\mu s \mathbf{F}_{\text {int }_{n}}$ and $\mathbf{M}_{t \square}^{*}=\mathbf{M}_{t \square}-\mu s \mathbf{M}_{n \square}$. If the matrix $\mathbf{M}^{*}$ is nonsingular, the second order differential system (59) is regular and the normal displacements 
of the free contact candidate nodes, as well as the tangential velocities and the normal reactions of the nodes in sliding contact, depend continuously on the initial conditions and on time. Consequently, in a sufficiently small neighborhood of the steady sliding state, the solution of (59) (and (58)) for perturbed initial conditions arbitrarily close to $\mathbf{U}_{f}^{0}, \mathbf{U}_{t}^{0}$ and $\dot{\mathbf{U}}_{f}^{0}=\dot{\mathbf{U}}_{t}^{0}=0$ leads to a "smooth" solution of the general system (40), (41), (44). The equations of motion (59) are linearized :

$$
\mathbf{M}^{*} \delta \ddot{\mathbf{U}}^{*}(t)+\mathbf{K}^{*} \delta \mathbf{U}^{*}(t)=\mathbf{0}
$$

where $\delta \mathbf{U}^{*}(t)$ denotes a small increment of the active displacements, $\mathbf{K}^{*}$ a nonsymmetric friction dependent tangent stiffness matrices of dimension $N^{*}(\leq N)$ constructed as $\mathbf{M}^{*}$. The main important feature is that if the steady sliding equilibrium state is dynamically unstable for the linearized system (60), it is also unstable for the non-linear system (59) and for the original system (40), (41), and (44). The analysis of the dynamic stability of the system (60) leads classicaly to the generalized eigenproblem :

$$
\left(\lambda^{2} \mathbf{M}^{*}+\mathbf{K}^{*}\right) \mathbf{V}^{*}=0 .
$$

The occurrence of a nontrivial eigenvector $\mathbf{V}^{*}$ for some $\lambda$ with a strictly positive real part implies the instability of the steady sliding state: a divergence instability if $\operatorname{Im}(\lambda)=0$ (non-oscillatory), a flutter instability if $\operatorname{Im}(\lambda) \neq 0$ (oscillatory).

The generalized eigenproblem (61) is solved by using the Lanczos algorithm ${ }^{21}$ along with the double QR method.

\section{SQUEAL FOR A RUBBER/GLASS CONTACT}

\subsection{The waist seal problem}

This application adresses the study of the noise generated by the waist seal of the lateral door of a car. This system is fixed to an internal metal sheet and is pressed against the window. The longitudinal and transversal sections of the door are schematically represented in figure 2 .

In some cases, this system induces squeal noise. The simulation is conducted on two real waist seals. The first one gives rise to squeal problems and is not used any longer. The second waist seal is a modified version of the first one that has completely removed the noise.

In subsection 6.2, the capabilities of the numerical model are presented on a detailled study of the first waist seal when the glass moves downward. Then, in subsection 6.3 , the model is used to compare the behavior of the two waist seals in terms of the possible occurrence of instabilities and of their consequences. 


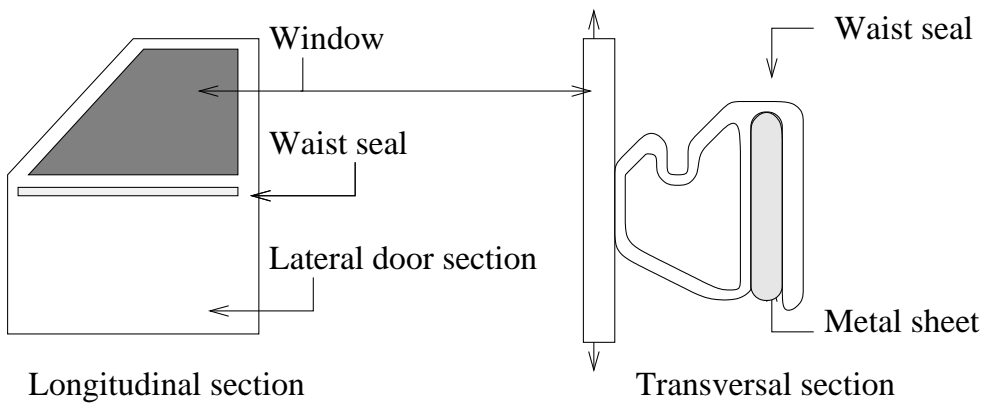

Figure 2: Schematic description of the lateral door.

\subsection{Simulation of a waist seal and stability analysis}

The basic parameters for the computation of the waist seal behavior are the geometry, the "loading", the material properties and the coefficient of friction. The geometry is plotted in grey on figure 3. The mesh is composed of 649 nodes and $538 \mathrm{Q} 4 \mathrm{P} 0$ finite elements. It has been particulary refined on the contact zone to ensure a minimum of 10 contact nodes. The black triangles $\mathrm{cm}$ mark the fixed boundary conditions (only a part of the waist seal is modelled) and the thin rectangle is a schematic representation of the glass window. The loading is applied in two steps : firstly a normal displacement of the obstacle is prescribed and the corresponding equilibrium position is computed for $\mu=0$. Then the value of the friction coefficient is successively increased, the corresponding steady sliding equilibrium positions being computed for an imposed tangential velocity of the obstacle $(80 \mathrm{~mm} / \mathrm{s})$ either upward or downward. The glass window is supposed to be plane and rectangular and the waist seal to be in a state of plane strain. The Mooney-Rivlin material coefficients are $a_{1}=0.293 \mathrm{MPa}$ and $a_{2}=0.177 \mathrm{MPa}$ and the bulk modulus is of $1200 M P a$. The experimental range of the coefficient of friction is $[0.1,0.8]$. This range has been obtained from experiments on small parallelepipedic samples of the waist seal material (with or without varnish) and for various interface conditions (with or without water).

The fixed point algorithm of section 4 is used to compute the steady sliding solutions. Computations are conducted with various friction coefficients (increments of 0.05). The deformed configuration after the initial normal loading is plotted in black on figure 3. The steady sliding configuration obtained for $\mu=0.4$ is plotted on figure 4 .

For the stability analysis, the eigenvalue spectrum of $\mathbf{M}^{*-1} \mathbf{K}^{*}$ is computed for all the steady sliding positions previously obtained. A divergence instability would be characterized by a negative real eigenvalue : it never occurs. Complex eigenvalues correspond to flutter instability. The analysis focuses on the occurrence of instabilities with frequencies belonging to the acoustic range, but some results are given for a larger range. 


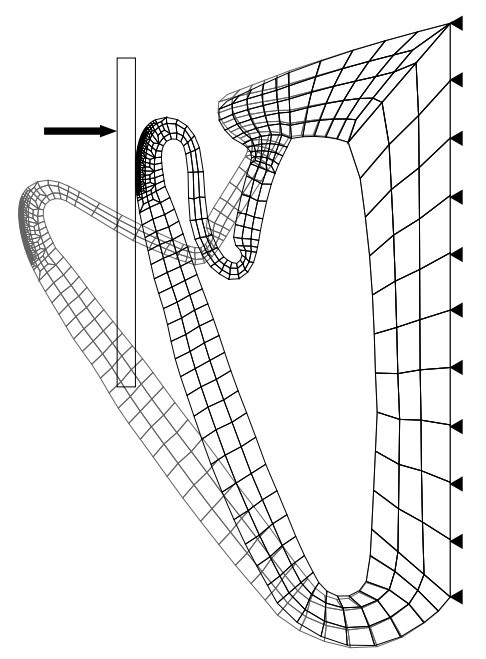

Figure 3: Initial normal loading, wiper compression with $\mu=0$.

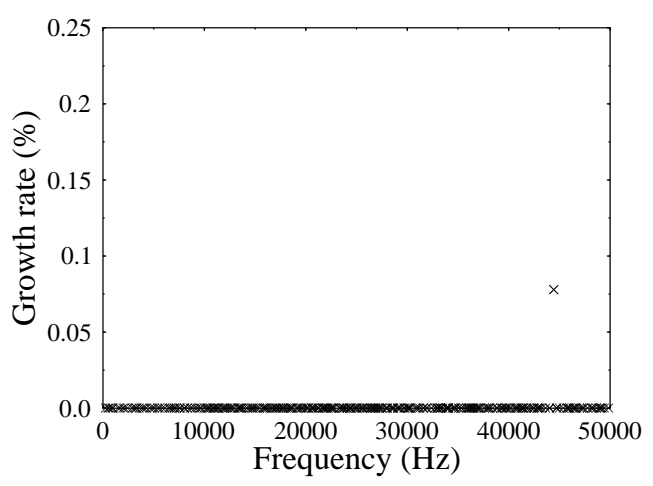

Figure 5: Eigenvalue spectrum of $\mathbf{M}^{*-1} \mathbf{K}^{*}, \mu=0.2$.

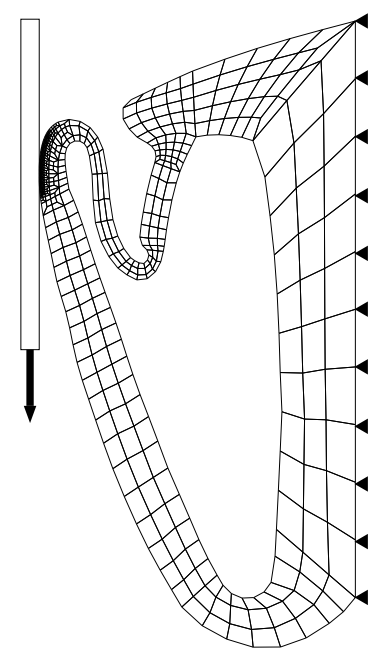

Figure 4: Glass downward: steady sliding position for $\mu=0.4$.

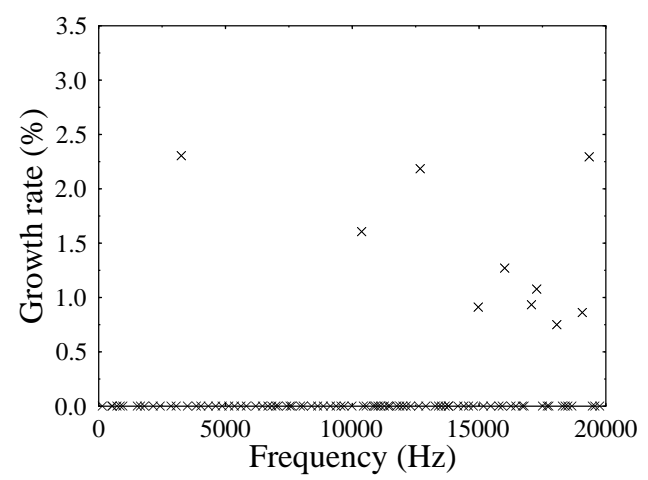

Figure 6: Eigenvalue spectrum of $\mathbf{M}^{*-1} \mathbf{K}^{*}, \mu=0.4$.

The first complex eigenvalue appears for $\mu=0.2$. The eigenvalue spectrum is plotted in figure 5. The eigenvalue spectrum of $\mathbf{M}^{*-1} \mathbf{K}^{*}$ obtained with $\mu=0.4$ is presented in figure 6 .

For $\mu<0.285$, the frequency of the lowest complex eigenvalue is high (about $44000 \mathrm{~Hz}$ ). On figure 7 , the coallescence for $\mu=0.285$ of two real eigenvalues is shown (frequency about $3250 \mathrm{~Hz}$ ). On the second plot of figure 7 , the growth rate of the flutter oscillations is plotted. This complex eigenvalue frequency remains close to $3250 \mathrm{~Hz}$ for the rest of the range of the friction coefficient.

It can be noted that the frequency associated to the lowest complex eigenvalue is relatively separated from the other ones. The associated eigenmode gives the spatial form of the growing dynamic solution close to the steady sliding position. On figure 8, the deformed configuration is plotted in grey and the deformed configuration plus the 
eigenmode multiplied by an amplification coefficient is plotted in black.
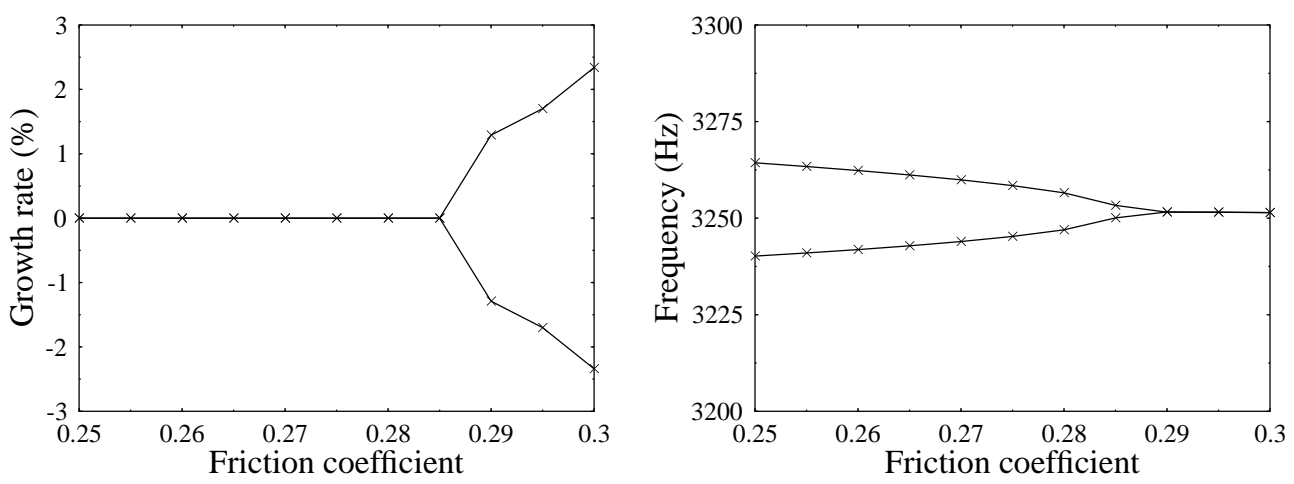

Figure 7: Coupling of two real eigenvalues, $\mu \in[0.25: 0.3]$.

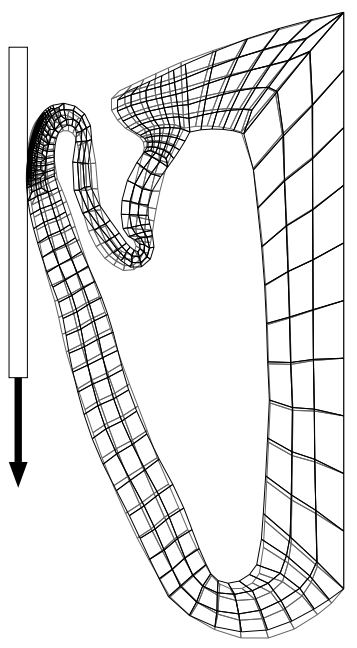

Figure 8: Eigenmode associated to the first flutter frequency, $\mu=0.4$, first version.

The dynamic behavior is computed by solving the dynamical problem P4 (see (53)) taking the steady sliding position and zero velocity as initial conditions. The only initial perturbation is due to the numerical precision in introducing those initial conditions. A slight amount of numerical dissipation is also introduced $\left(\theta_{1}=\theta_{2}=0.55>0.50\right)$.

The evolution of the global contact forces is plotted on figure 9. During a short time interval, the contact forces seem to remain constant (with $P_{t}=\mu P_{n}$ ). Then, growing oscillations with the flutter frequency $(3250 \mathrm{~Hz})$ are perceptible. About $0.25 \mathrm{~s}$ after the beginning of the dynamical computation, the sliding of some contact nodes becomes intermittent. About 0.03s later, this stick-slip becomes global and is accompanied by 


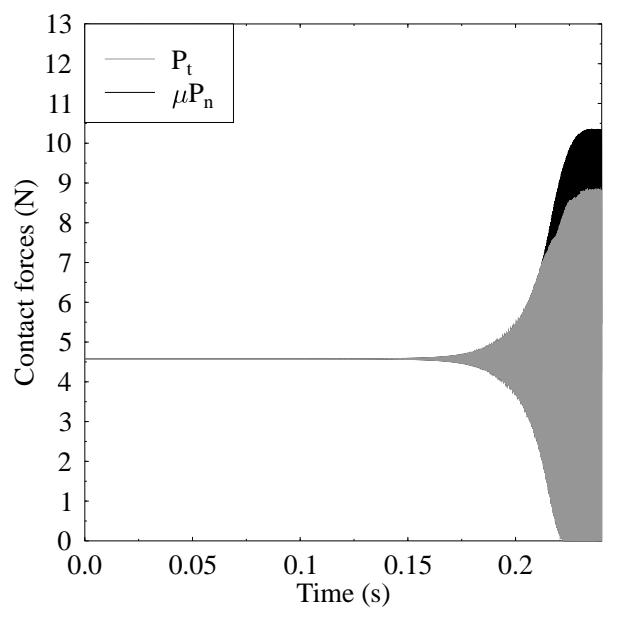

Figure 9: Evolution of the contact forces for $\mu=0.4$.

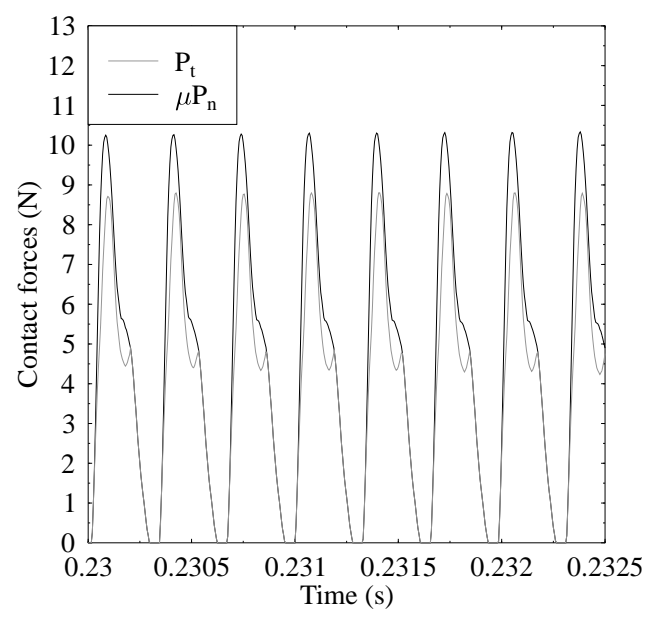

Figure 10: Evolution of the contact forces for $\mu=0.4$, time interval of $0.2510^{-2} \mathrm{~s}$.

global losses of contact (see figure 10). It can be noted on figure 10 that the evolution has reached what looks like a limit cycle. The amplitude of the oscillations of the contact forces is very large and the waist seal hammers the window with a frequency that belongs to the acoustic range. For this computation of the dynamical behavior of the waist seal, the perturbation introduced is very small (only the numerical precision) and a relatively long time is necessary to reach the limit cycle. This time may be reduced by introducing a slightly larger initial perturbation. We have checked that for different forms of this extra small perturbation, the same limit cycle is attained.

For a larger coefficient of friction $(\mu=0.8)$, the growth of the flutter oscillations is larger in the sense that these oscillations become significant for $\tau=0.045 \mathrm{~s}$ (instead of $\tau=0.15 \mathrm{~s}$ for $\mu=0.4$ ). The phenomenon is more complex as presented on figure 11. As previously, global losses of contact occur during the oscillations.

An non-linear elastic behavior has been assumed in the theorical model adopted for the waist seal. In reality, this kind of rubberlike material is viscoelastic, and some small numerical damping has been always considered in our computations, by means of the values adopted for $\theta_{1}$ and $\theta_{2}$. It is interesting to check the effect of an increase of the numerical damping on the solution. Using $\theta_{1}=\theta_{2}=0.60$ instead of $\theta_{1}=\theta_{2}=0.55$ leads to the results presented on figure 12. The nature of the phenomenon is still the same with an oscillation frequency equal to $3250 \mathrm{~Hz}$, but the evolution is more regular and no global losses of contact are observed. This absence of loss of contact has been also observed with $\mu=0.4$ when $\theta_{1}=\theta_{2}=0.60$.

We give now some further details of the dynamic evolution in this case $(\mu=0.8$ 


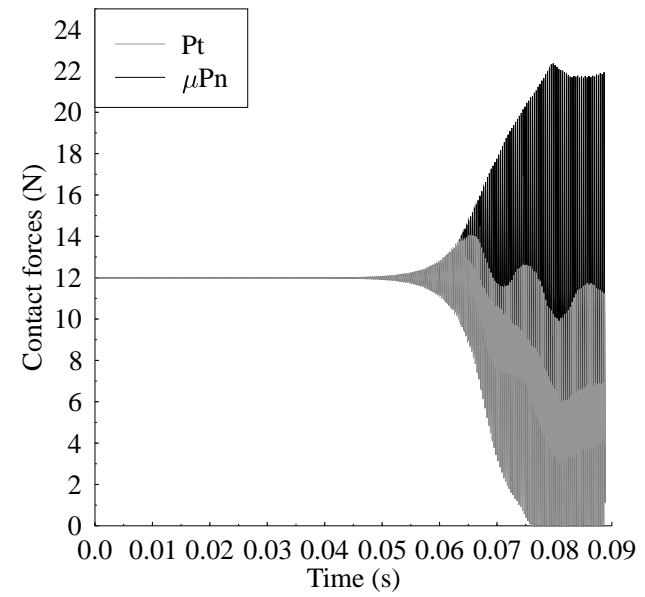

Figure 11: Evolution of the contact forces for $\mu=0.8, \theta_{1}=\theta_{2}=0.55$.

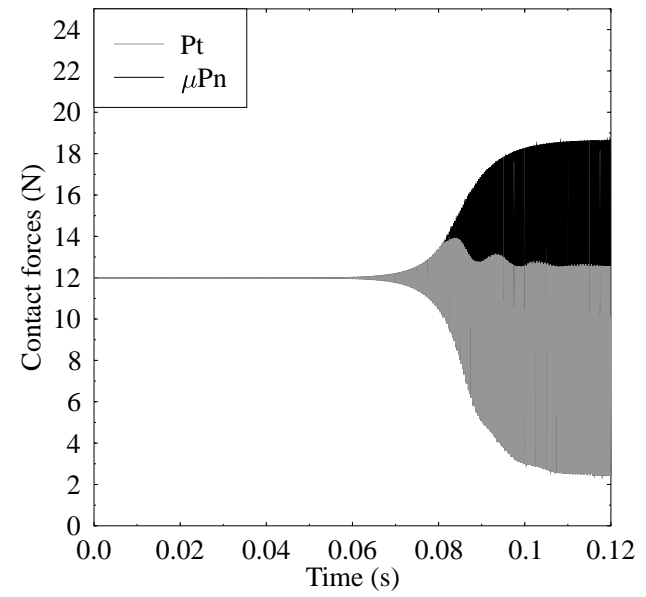

Figure 12: Evolution of the contact forces for $\mu=0.8, \theta_{1}=\theta_{2}=0.60$.

and $\left.\theta_{1}=\theta_{2}=0.60\right)$. We present first the evolution of the tangential velocity of all the contact nodes on a short time interval $(\tau \in[0.25 s ; 0.2505 s])$ on figure 13. As expected, a stick-slip phenomenon is observed but it should be noted that it is more complex than a simple succession of global stick and global slip states : the contact nodes do not start to slip simultaneously. We focus now on the the normal and tangential movements of a node located on the edge of the contact zone (node 1 on figure 13). Phase plane plots of that tangential movement are presented in figure 14 (the complete evolution) and in figure 15 (the "limit cycle"). The normal component is presented on figure 16. It can be noted that even for $\theta_{1}=\theta_{2}=0.60$, the "limit cycle" oscillation has a short period of loss of contact; for example, one cycle of the node 1 involves the following sequence of states : stick, slip, loss of contact, slip and back to stick. The results obtained with the window moving upward are very similar to the previous one, but with a lower flutter frequency (about 1980Hz).

\subsection{Comparison of the analysis for two different waist seals}

Because of the lack of detailed experimental results, the only way to give a rough validation of the model has been to compare the results of the stability analyses of two waist seals, one of which is known to be noisy and the other one to be "silent". Those two waist seals are flocked, and with this flock the friction coefficient is experimentally found to belong to $[0.15,0.45]$. The waist seal simulated in the previous subsection was known to be noisy. We will conduct now the analysis of the modified "silent" geometry. This second geometry is shown to be less prone to flutter instabilities than the first one. The new geometry is given on figure 17 . 


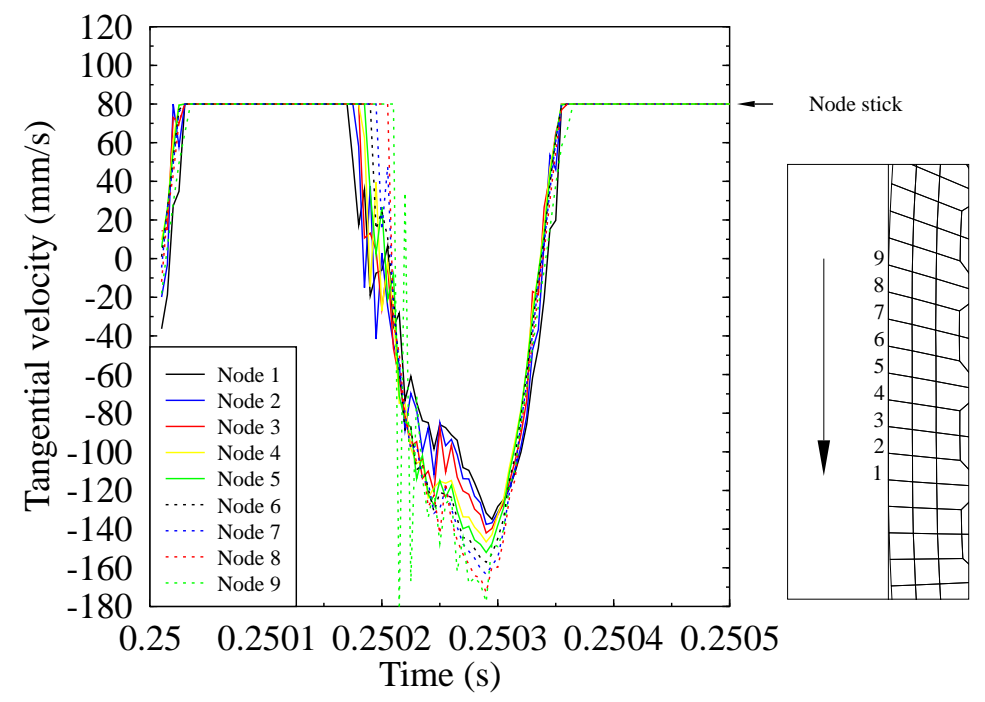

Figure 13: Evolution of the tangential velocity of the contact nodes with $\tau \in[0.25 \mathrm{~s} ; 0.2505 \mathrm{~s}]$, the window velocity is of $80 \mathrm{~mm} / \mathrm{s}$.

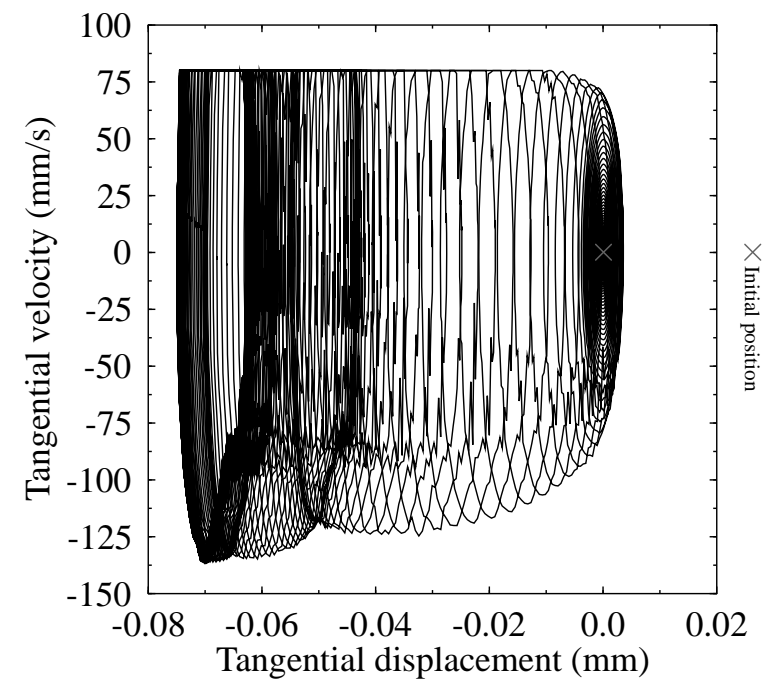

Figure 14: Tangential velocity of node 1, time interval : $\tau \in[0 ; 0.25]$.

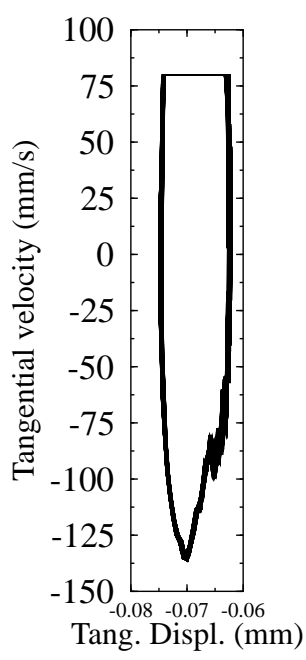

Figure 15: Limit cycle, $\tau \in$ $[0.2 ; 0.25]$. 


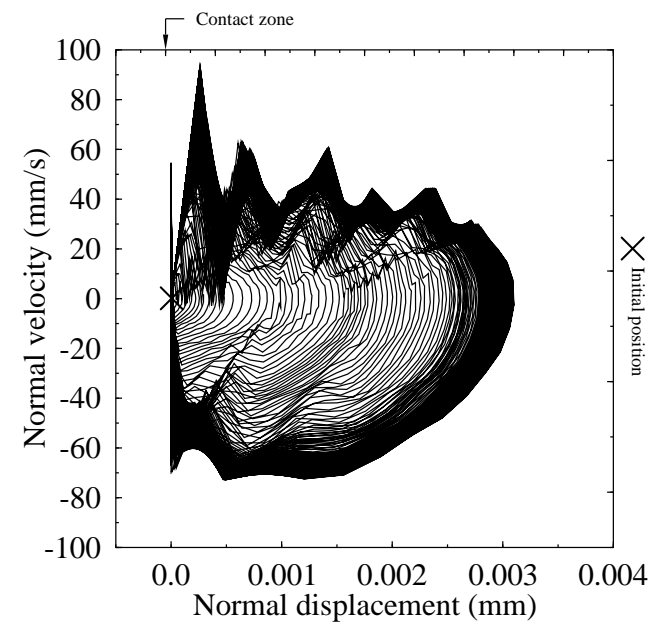

Figure 16: Normal components of node 1, time interval $: \tau \in[0 ; 0.25]$.

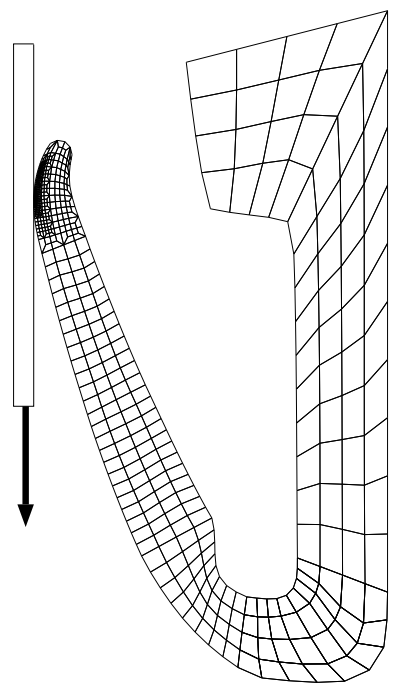

Figure 17: Steady sliding position, window downward, $\mu=0.4$, second waist seal.

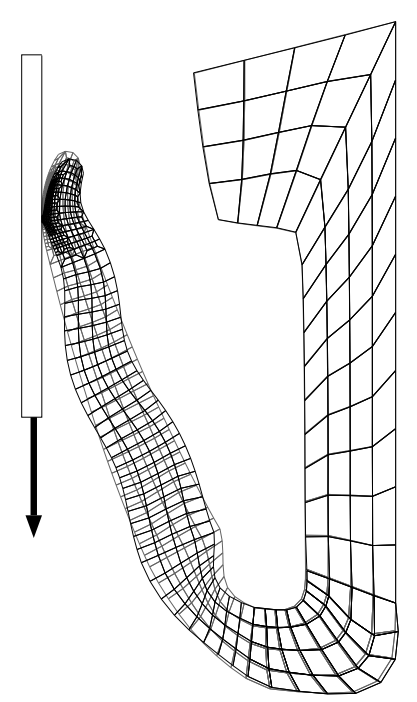

Figure 18: Eigenmode associated with the lowest flutter frequency, $\mu=0.4$, second waist seal.

6.3.1 Window moving upward $\left(\mu=0.4\right.$ and $\left.\theta_{1}=\theta_{2}=0.55\right)$

The eigenvalue spectra of $\mathbf{M}^{*-1} \mathbf{K}^{*}$ for the upward movement of the window with $\mu=0.4$ are presented in figure 19 (first geometry) and in figure 20 (second geometry). The eigenmode associated to the lowest flutter frequency is plotted on figure 18. For the first case, the frequency corresponding to the complex eigenvalue is of $1950 \mathrm{~Hz}$ with a growth rate of $3.5 \%$ and for the second case, it is of $18890 \mathrm{~Hz}$ with a growth rate of $0.06 \%$. For the second geometry, squeal noise is not expected, because, for the computed high frequency of flutter (which in any case would not be in the acoustic range) and for the corresponding 


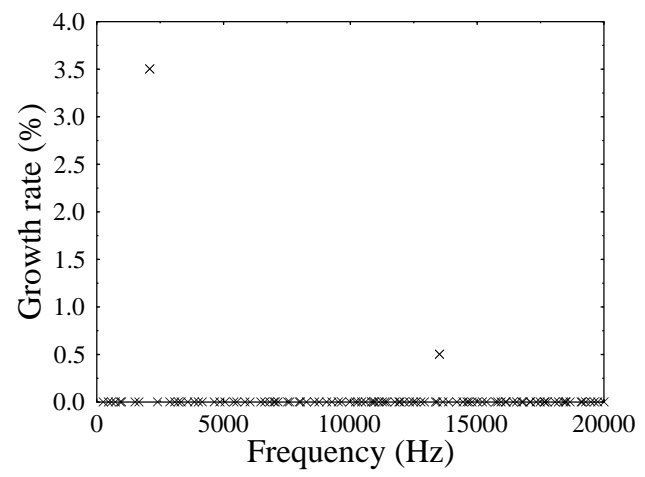

Figure 19: Eigenvalues spectrum of $\mathbf{M}^{*-1} \mathbf{K}^{*}, \mu=0.4$, window moving upward, first waist seal.

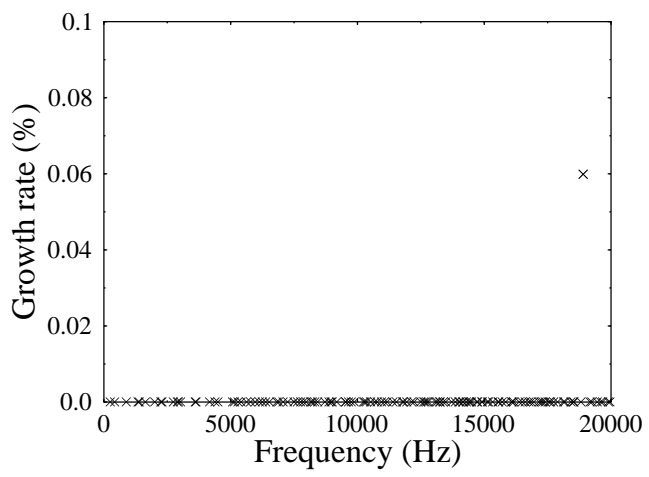

Figure 20: Eigenvalues spectrum of $\mathbf{M}^{*-1} \mathbf{K}^{*}, \mu=0.4$, window moving upward, second waist seal.

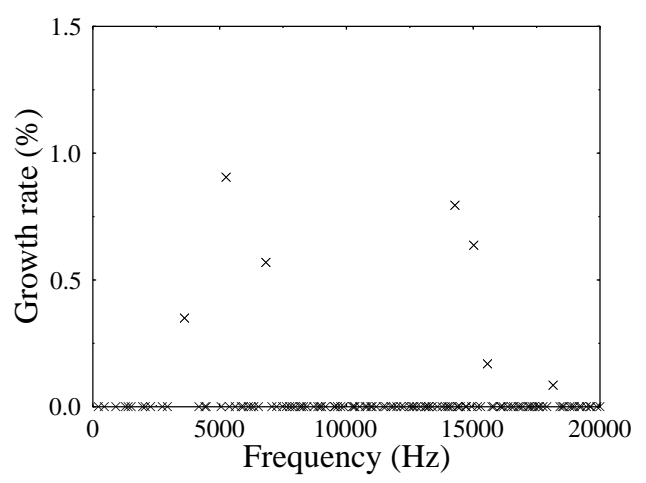

Figure 21: Eigenvalues spectrum of $\mathbf{M}^{*-1} \mathbf{K}^{*}, \mu=0.4$, window moving downward, second waist seal.

small growth rate, one may expect that any small amount of material dissipation will cancel out the instability.

\subsubsection{Window moving downward $\left(\mu=0.4\right.$ and $\left.\theta_{1}=\theta_{2}=0.55\right)$}

For the window moving downward, the eigenvalue spectrum of $\mathbf{M}^{*-1} \mathbf{K}^{*}$, corresponding to the first case has been given on figure 6 . The one corresponding to the second waist seal is given on figure 21. The frequencies corresponding to the first complex eigenvalue are similar in both cases $(3250 \mathrm{~Hz}$ for the first geometry, $3050 \mathrm{~Hz}$ for the second one) but the growth rate is six times smaller for the second geometry ( $0.35 \%$ instead of $2.4 \%$ ). The second case involves also two other frequencies in the acoustic range $(5240 \mathrm{~Hz}$ and $6810 \mathrm{~Hz}$ ) but their growth rates are also smaller than the one of the first geometry. 


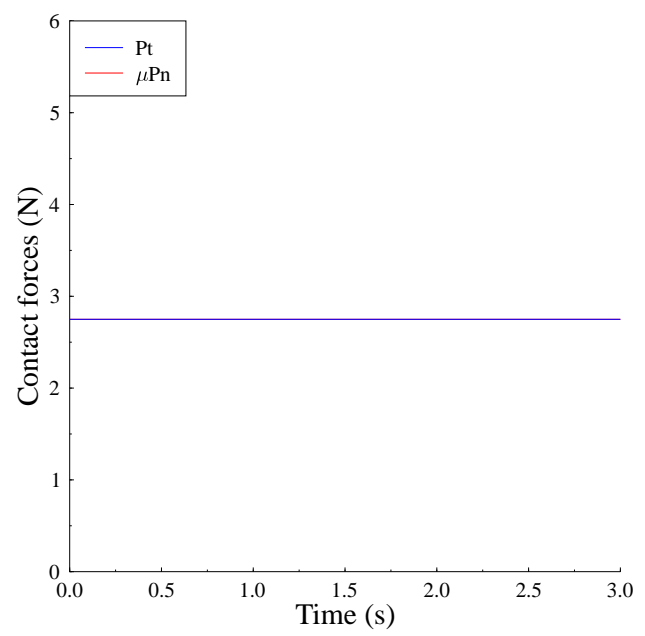

Figure 22: Evolution of the contact forces for $\mu=0.4, \theta_{1}=\theta_{2}=0.55$, second waist seal.

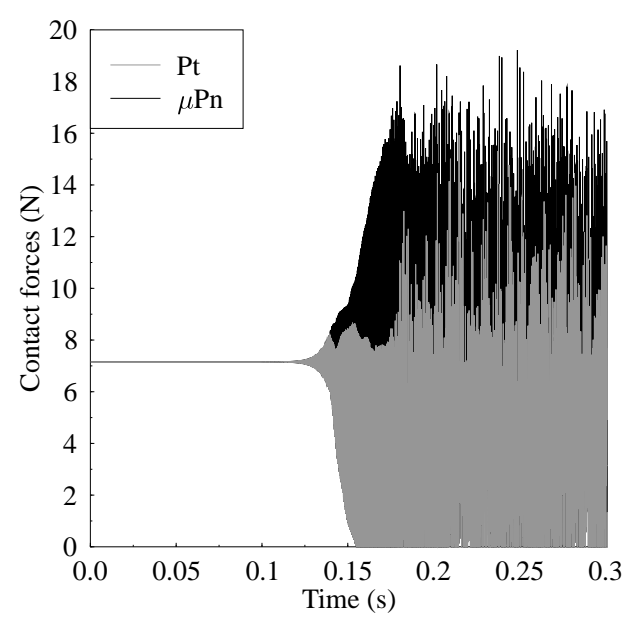

Figure 23: Evolution of the contact forces for $\mu=0.8, \theta_{1}=\theta_{2}=0.55$, second waist seal.

To check the possible consequences of flutter in this second case, dynamic analyses were performed with the numerical parameters used earlier to obtain figure 22 (namely $\left.\theta_{1}=\theta_{2}=0.55\right)$. The result given on figure 22 shows that no oscillating solution emerges.

The first geometry is more prone to flutter instability then the second one and, using the same material and numerical parameters, important oscillations are numerically obtained for the first "noisy" geometry whereas no oscillations occur in the numerical tests with the second "silent" geometry.

For sufficiently large friction coefficient (for $\mu=0.8$ ) the second geometry may also lead to squeal (the first one was unstable for both $\mu=0.4$ and $\mu=0.8$ ). The lowest flutter frequency obtained with $\mu=0.8$ is of $2965 \mathrm{~Hz}$ and the corresponding growth rate is of $3.35 \%$. The dynamic solution plotted on figure 23 should be compared with the one obtained for the first geometry and given on figure 11 .

\section{ACKNOWLEDGEMENT}

Research supported by Renault in the project "Numerical study of the friction between a glass window and a wiper".

\section{References}

[1] G. Björkman, A. Klarbring, B. Sjödin, T. Larsson and M. Rönnqvist, 'Sequential quadratic programming for non-linear elastic contact problems', Int. J. Numer. Meth. Engng., 38, 137-165 (1995). 
[2] P. Chabrand, F. Dubois and M. Raous, 'Various numerical methods for solving unilateral contact problems with friction', Mathl. Comput. Modelling., 28, 97-108 (1998).

[3] R. W. Cottle, J. S. Pang and R. Stone, The linear complementarity problem, Computer Science and Scientific Computing, Academic press, New-York, 1992.

[4] P. J. Flory, 'Thermodynamic relations for high elastic materials', Trans. Faraday. Soc., 57, 829-838 (1961).

[5] M. S. Gadala, 'Numerical solutions of nonlinear problems of continua - II. A survey of incompressibility constraints and software aspects', Comput. Struct., 22, 841-855 (1986).

[6] Q. C. He, J. J. Telega and A. Curnier, 'Unilateral contact of two solids subject to large deformations: formulation and existence results', Proc. R. Soc. Lond. A, 452, 2691-2717 (1996).

[7] M. Jean and J.J. Moreau, 'Dynamics in the presence of unilateral contact and dry friction: a numerical approach', in Del Piero and Maceri (eds.), Unilateral problems in structural analysis - 2, CISM lectures vol. 304, Springer Verlag, 1987.

[8] M. Jean, 'The non smooth contact dynamics method', Comput. Meth. Appl. Mech. Engng., to appear.

[9] A. Klarbring, 'Contact, friction, discrete mechanical structures and mathematical programming', Lecture notes for the CISM course Contact Problems: Theory, Methods, Applications, Udine, to appear.

[10] A. Klarbring, 'Steady sliding and linear complementarity', in M. Ferris and J. C. Pang (eds.), Complementarity and variational problems: state of the art, SIAM publication, Philadelphia, 1997, pp. 132-147.

[11] A. Klarbring and G. Björkmann, 'Solution of large displacement contact problems with friction using Newton's method for generalized equations', Int. J. Numer. Meth. Engng., 34, 249-269 (1992).

[12] C. H. Liu, G. Hofstetter and H. A. Mang, '3d finite element analysis of rubber-like materials at finite strains', Engng. comput., 11, 111-128 (1994).

[13] J. A. C. Martins, J. T. Oden and F. M. F. Simões, 'A study of static and kinetic friction', Int. J. Engng. Sci., 28, 29-92 (1990).

[14] J. A. C. Martins and A. Pinto da Costa, 'Stability of finite dimensional systems with unilateral contact and friction: non-linear elastic behaviour and obstacle curvature', Report ICIST AI 9/98, Instituto Superior Técnico, Universidade Técnica de Lisboa, Lisboa, Portugal. 
[15] J. A. C. Martins, S. Barbarin, M. Raous and A. Pinto da Costa, 'Dynamic stability of finite dimensional linearly elastic systems with unilateral contact and Coulomb friction', Comput. Meth. Appl. Mech. Engng., to appear.

[16] F. Moirot, 'Etude de la stabilité d'un équilibre en présence de frottement de Coulomb, application au crissement des freins à disques', Ph.D. Thesis, Ecole Polytechnique, Paris, France, 1998.

[17] J. T. Oden, J. A. C. Martins, 'Models and computational methods for dynamic friction phenomena', Comput. Meth. Appl. Mech. Engng., 52, 527-634 (1985).

[18] S. Pandit and S. Deo, Differential systems involving impulses, Lectures notes in mathematics, Springer Verlag, 1982.

[19] E. B. Pires and L. Trabucho, 'The steady sliding problem with nonlocal friction', Int. J. Engng. Sci., 28, 631-641 (1990).

[20] P. Rabier, J. A. C. Martins, J. T. Oden and L. Campos, 'Existence and local uniqueness of solutions for contact problems with non-linear friction laws', Int. J. Engng. Sci., 24, 1755-1768 (1986).

[21] C. Rajakumar and C. Rogers, 'The Lanczos algorithm applied to unsymmetric generalized eigenvalue problem', Int. J. Numer. Meth. Engng., 32, 1009-1026 (1991).

[22] M. Raous and S. Barbarin, 'Stress waves in a sliding contact. Part 1 : modelling', in D. Dowson et al. (eds.), Proceedings of the $22^{\text {nd }}$ Leeds Lyon Symposium on Tribology, Elsevier Science, 1996, pp. 39-44.

[23] R. T. Rockafellar, Convex analysis, Princeton University Press, 1970.

[24] J. C. Simo et R. L. Taylor, 'Quasi-incompressible finite elasticity in principal stretches. Continuum basis and numerical algorithms', Comput. Meth. Appl. Mech. Engng., 85, 272-310 (1991).

[25] D. Vola, E. Pratt, M. Jean and M. Raous, 'Consistent time discretization for a dynamical frictional contact problem and complementarity techniques', Revue Européenne des Eléments Finis, 7, 149-162 (1998).

[26] D. Vola, A. Pinto da Costa, S. Barbarin, J.A.C. Martins and M. Raous, 'Bifurcations and instabilities in some finite dimensional frictional contact problems', in F. Pfeiffer (ed.), Proceedings of 1998 IUTAM symposium: unilateral multibody dynamics, Kluwer, to appear.

[27] T. Zeghloul and B. Villechaise, 'Stress waves in a sliding contact. Part 1 : experimental study', in D. Dowson et al. (eds.), Proceedings of the $22^{\text {nd }}$ Leeds Lyon Symposium on Tribology, Elsevier Science, 1996, pp. 33-37. 